\title{
Neue Ära in der Ernährung von Säuglingen
}

\section{Spezielle Kohlenhydrate der Muttermilch jetzt auch in Säuglingsmilchnahrung verfügbar.}

Humane Milch Oligosaccharide (HMO) sind spezielle Kohlenhydrate, die fast nur in Muttermilch vorkommen. Sie sind in der Muttermilch die drittgrößte Komponente nach dem Milchzucker und den Milchfetten. HMO werden ausschließlich in der Brustdrüse der Mutter nur während der Stillzeit gebildet. Erstmals ist es gelungen, einige dieser Kohlenhydrate so herzustellen, dass sie mit den HMO in Muttermilch strukturidentisch sind und somit in Säuglingsmilchnahrung eingesetzt werden können.

\section{Rezente wissenschaftliche Daten}

Neue Studien dokumentieren: Säuglingsmilch mit zugesetzten Humanen Milch Oligosacchariden ist sicher, gut verträglich und unterstützt das altersgerechte Wachstum.

Dr. med. Mike Poßner, medizinischer Leiter des Nestlé Nutrition Institutes, prä-

Literatur bei der Redaktion sentierte anlässlich eines Expertentalks im August 2018 in Wien die ersten Ergebnisse: „Wir haben die ersten Studien vorliegen, in denen wir die Bedeutung von HMO in Muttermilch und deren Wirkung auf das Mikrobiom des Säuglings untersucht haben. Wir stehen somit am Start einer spannenden Reise in die Zukunft und werden den Zusatz von HMO in Säuglingsmilchnahrung im Rahmen von klinischen Studien weiter untersuchen", so der Humanmediziner (• Abb. 1).

\section{HMO für die gesunde Entwicklung des Säuglings}

In der Forschung liegt zunehmend Evidenz vor, dass HMO für eine gesunde Entwicklung des Säuglings wichtig sind. Diese betreffen:

- die Förderung des Wachstums bestimmter nützlicher Bifidusbakterien und die Hemmung schädlicher Bakterien.

- HMO können als „Andockstationen“ für Bakterien und Viren, die

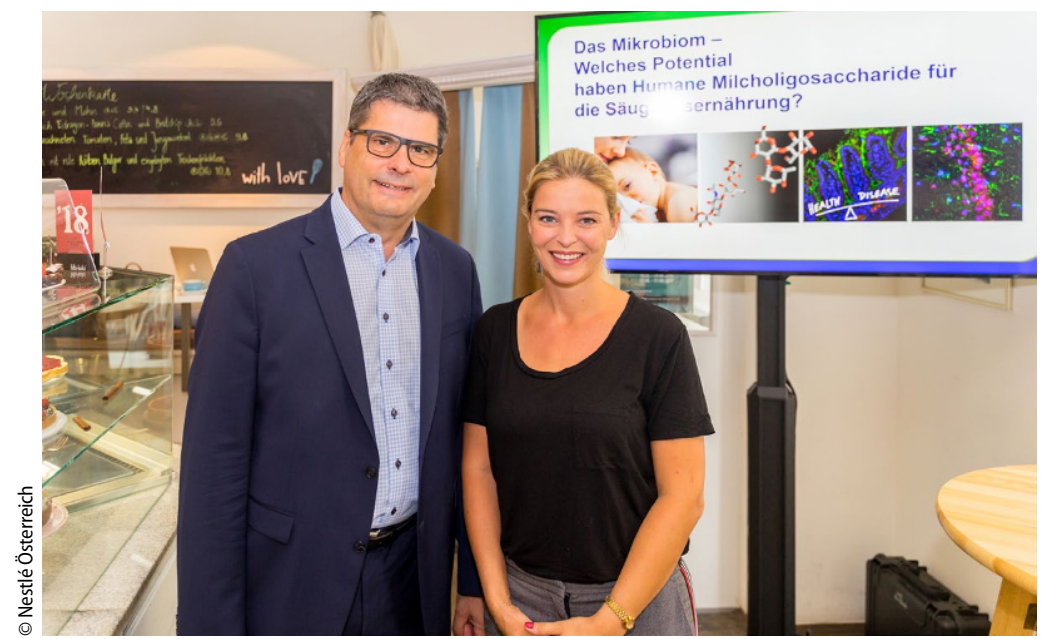

Abb. 1 A Dr. med. Mike Poßner, medizinischer Leiter des Nestlé Nutrition Institutes im Expertentalk mit Dr. med. Christine Reiler (ORF)
Krankheiten verursachen, angesehen werden.

- die Stärkung der DarmbarriereFunktion.

- 1 bis $2 \%$ der HMO gelangen unverändert in den Blutkreislauf und zeigen eine potentielle Wirkung auf das Immungleichgewicht.

\section{Studien mit HMO in Säuglings- milchnahrung}

Neue Studien dokumentieren: Säuglingsmilch mit zugesetzten $\mathrm{HMO}$ ist sicher, gut verträglich und unterstützt das altersgerechte Wachstum. Eine erste Studie zeigt, dass die Zugabe von HMO ein Mikrobiom ähnlich dem beim gestillten Säugling fördert. Außerdem weisen Daten darauf hin, dass bestimmte Erkrankungen - insbesondere Bronchitis - seltener auftreten. Auch der Einsatz von Antibiotika und fiebersenkenden Medikamenten ist niedriger. Dies alles sind Kriterien, die auf ein gut funktionierendes Immunsystem hinweisen.

Aktuell werden $\mathrm{HMO}$ in Österreich ausschließlich in einer Säuglingsmilchnahrung von Nestlé eingesetzt. Es handelt sich um 2'FL und LNnT: Diese machen bei der Mehrzahl der Frauen mehr als $40 \%$ der HMO in der Muttermilch aus. Beide HMO werden sowohl von der EFSA in Europa als auch der FDA in den USA als sicher bewertet.

\section{Weitere Informationen:}

www.nestlenutrition-institute.de

Paediatr. Paedolog. Austria 2019 · 54:48 https://doi.org/10.1007/s00608019-0647-0

(c) Springer-Verlag GmbH Austria, ein Teil von Springer Nature 2019 